Journal of Innovative Image Processing (JIIP) (2019)

Vol.01/ No. 01

Pages: 51-60

https://www.irojournals.com/iroiip/

DOI: https://doi.org/10.36548/jiip.2019.1.006

\title{
A REVIEW ON IOT BASED MEDICAL IMAGING TECHNOLOGY FOR HEALTHCARE APPLICATIONS
}

\author{
Dr. Abraham Chandy, \\ Professor, \\ Karunya University, \\ Coimbatore, India. \\ Email id: abrahamchandy@karunya.edu
}

\begin{abstract}
The usage of the IOT technology in the healthcare applications ensures the health care industries to enhance the quality of the care and minimize the expense with the automation and the resource optimization provided by it. The IOT in the medical imaging enables to have identification and the corrective measures to be taken in the real time with the auto analyzing of the imaging apparatus parameters at ease. Digitization reaching to many areas in the medical technology has also paved its way for the monitoring and the management of the medical equipment's, the internet of things in the medical imaging would reduce the waiting time and the vexation for the patients as well as the physicians. So the paper presents the review on the internet of things based medical imaging technology for the health care applications providing the significance of the internet of things in the medical imaging technology.
\end{abstract}

Keywords: Internet of Things, Medical Imaging, Health Care, Automation and Resource Optimization.

\section{INTRODUCTION}

Among a wide range of applications that are enabled using the internet of things the health care applications involving the IOT have become more significant [7] as they minimize the cost and the weariness caused for the patients along with the concurrent improvements in the outcomes. The Internet of things incorporated into the health care industry would also allow having an efficient and an easy management and monitoring. The author Sapna et al [8] from her view describes the internet of things as the revolution in the healthcare avoiding the lack or the delay in the diagnosis, failures in the appropriate testing or the initiation in the follow up, the insufficiency to access the medical history of the patients, misconception and the wrong prescriptions, lack of knowledge about the person previous medical records. The author S. Smys et al in his paper [15] and [16] elaborates the topology of WSN in the IOT, and the applications and the protocols of the IOT. The latest development in the sensors also plays a significant role in internet of things for the medical industry; as they are enable an easy access and the analyses of medical records that were difficult to access before. The fig.1 below shows the IOT in the health care.

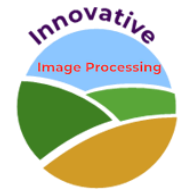


Journal of Innovative Image Processing (JIIP) (2019)

Vol.01/ No. 01

Pages: 51-60

https://www.irojournals.com/iroiip/

DOI: https://doi.org/10.36548/jiip.2019.1.006

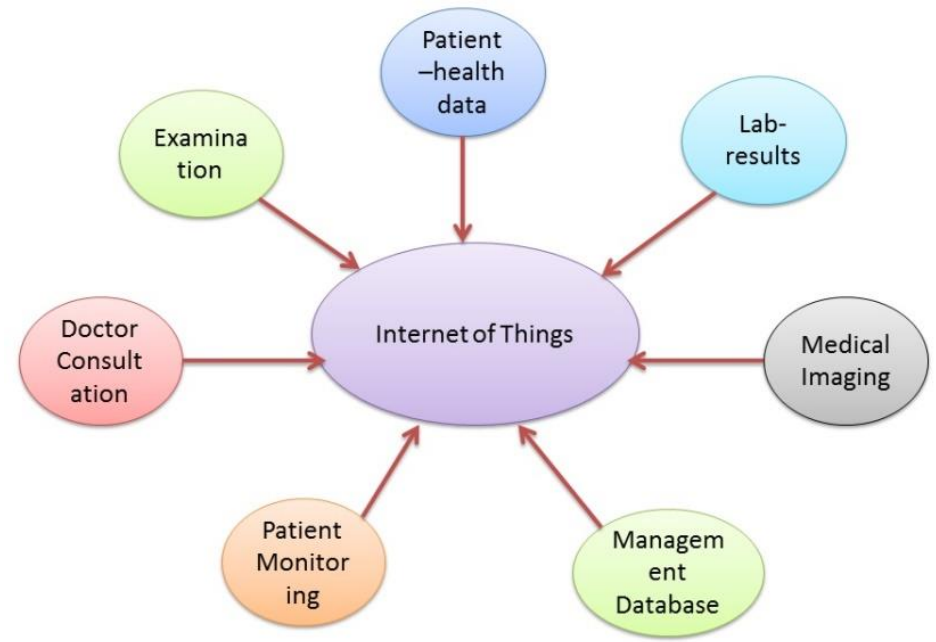

Fig .1 Internet of Things in Health Care

The paper specifically concentrates on the review of the importance of the IOT in the medical imaging. Medical imaging refers to the acquiring of the human body internal images for the diagnosis of the disease or treatment or research. The medical imaging is one of the vital requirements in the hospital. The internet of things in the medical imaging enables the sharing, diagnosis and the retrieval of information in the real time and eludes the problem of repeated examinations, consultation difficulties and the misdiagnosing.

The paper elaborates the review of incorporating Internet of Things with the medical imaging equipment's for the easy sharing of the images over the internet result in more accurate diagnosis and the treatment in real time.

The rest of the paper is arranged with the review in 2 sources of the medical imaging for the diagnosing of various diseases and involvement of the IOT in the medical imaging in 3 and the significance of the IOT in the health care industry 4. And conclusion in 5.

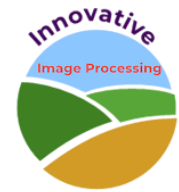


Journal of Innovative Image Processing (JIIP) (2019)

Vol.01/ No. 01

Pages: 51-60

https://www.irojournals.com/iroiip/

DOI: https://doi.org/10.36548/jiip.2019.1.006

\section{DIFFERENT SOURCES OF MEDICAL IMAGING}

The machine to machine communications provided by the internet of things, assists the medical imaging equipment's to enhance the quality and minimize the expense of the operations. This section includes the different sources of the medical imaging and the involvement of the IOT in the medical imaging in the disease diagnosis.

In this section the few sources of the medical imaging available in the medical industry for the diagnosis of the disease are elaborated.

\subsection{MRI (MAGNETIC RESONANCE IMAGING)}

The Magnetic Resonance imaging utilizes the computer the powerful magnetic field, and the radio frequency pulses to generate clarified pictures of the organs in the human body, such as bones, soft tissues and the structure of the internal body. Khoo et al [12] in his research has put forward the usage for the magnetic resonance imaging its consideration and its application in the radiotherapy treatment. It is also referred as the nuclear MRI or the magnetic resonance tomography and is used in the diagnosis for the brain tumors, trauma to the head or brain, problems in spine, heart joints and bones, abnormalities in the liver, uterine, abdomen and the brain. The MRI enables one to have the superior images of our internal body. The fig. 2 below is the image of the MRI scanner.

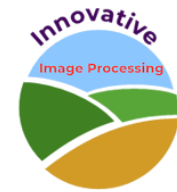


Journal of Innovative Image Processing (JIIP) (2019)

Vol.01/ No. 01

Pages: 51-60

https://www.irojournals.com/iroiip/

DOI: https://doi.org/10.36548/jiip.2019.1.006

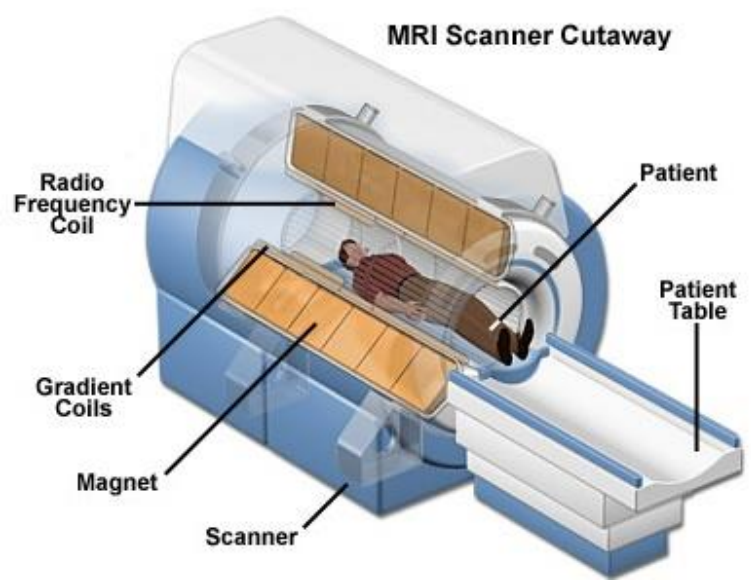

Fig .2 MRI Scanners

\subsection{X-RAY (X-RADIATION)}

The X-Ray are used for the capturing clear and the accurate images of the using the radiation. They use the electromagnetic radiation to penetrate into the structure to create images of the structure and found helpful in the diagnosing and detecting the abnormalities within the body. Kieffer et al [10] in his paper provides the utilization of the X-Rays in the medical imaging The fig. 3 below shows the image of the X-Ray.

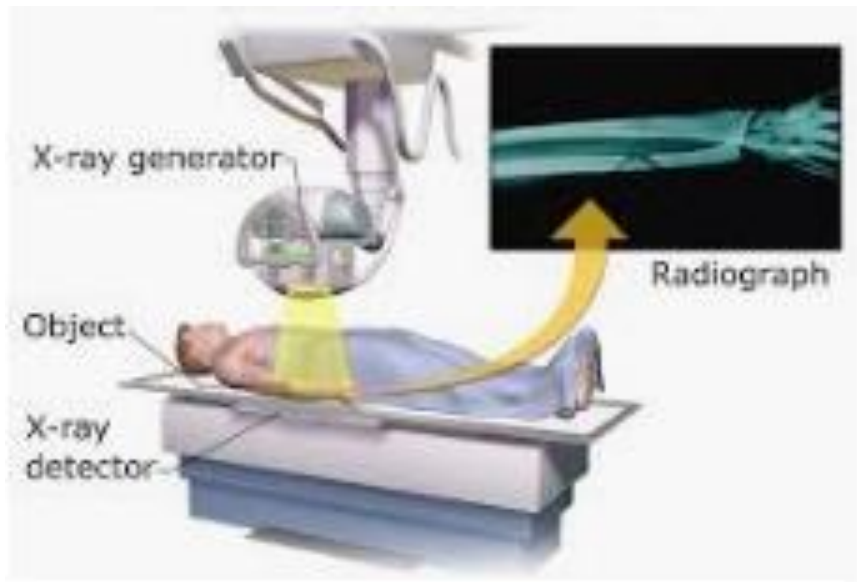


Journal of Innovative Image Processing (JIIP) (2019)

Vol.01/ No. 01

Pages: 51-60

https://www.irojournals.com/iroiip/

DOI: https://doi.org/10.36548/jiip.2019.1.006

Fig.3 X-Radiation

\subsection{CT (COMPUTED TOMOGRAPHY)}

The CT utilizes the X-Rays to have a detailed picture of the organs of the human body in very thin slices. The author Zinreich et al [13] describes the essentiality of the computed tomography in the medical imaging he particularly concentrated on the medical imaging through the CT for the endoscopic surgery. The fig. 4 below shows the CT scan that is utilized in the medical imaging.

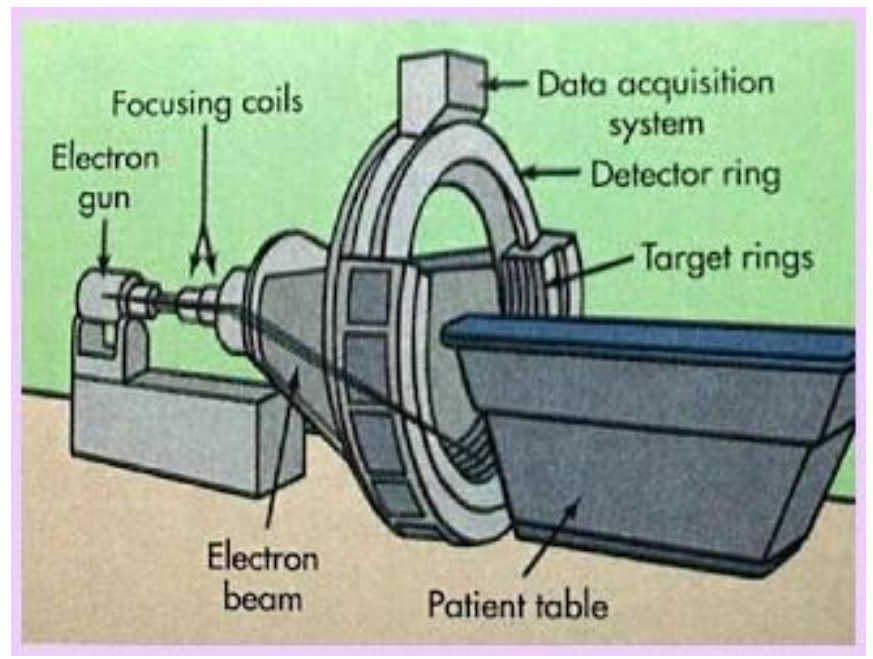

Fig.4 CT Scan

\subsection{ULTRA SOUND}

The ultra sound scans are the medical imaging equipment utilized for the having a look on the inside of the body. They are the acoustic energy in the form of the waves with the frequency higher than the human hearing range and used in the analyzing the inside of the human body as proffered by the Nikolov, et al [11] it finds its necessity in the medical imaging of the abdominal problems, uterus, the kidney problems and in the identifying the status of the baby in the fetus etc. the fig. 5 below shows the ultra sound scanner equipment.

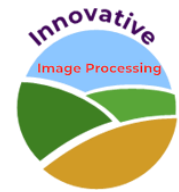


Journal of Innovative Image Processing (JIIP) (2019)

Vol.01/ No. 01

Pages: 51-60

https://www.irojournals.com/iroiip/

DOI: https://doi.org/10.36548/jiip.2019.1.006

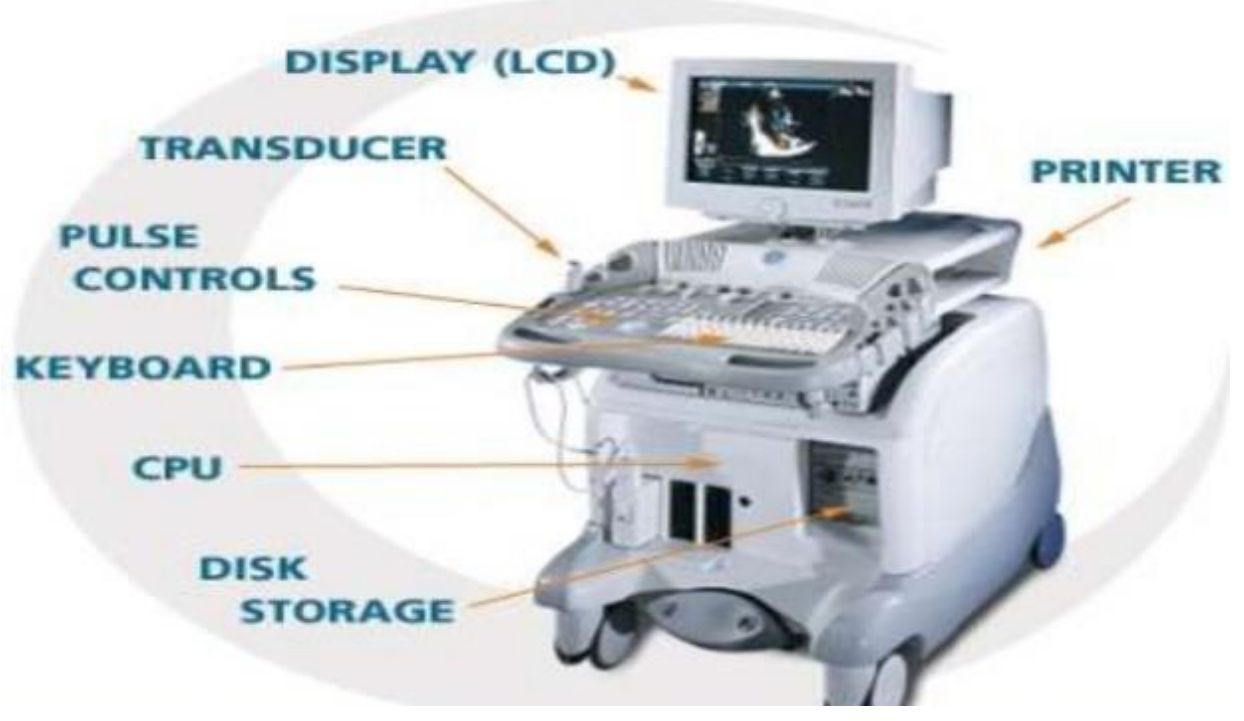

Fig .5 Ultra Sounds

The above section shows the available medical imaging equipment's that are used for the diagnoses or in the detection of the disabilities, abnormalities or disease in the human body. Though the equipment's seem to be very effective in the disease diagnosis the major problem prevailing nowadays is the increased number of patients and the increased number of the reports that leads to confusion, misplacing of the records and the wrong treatments so to avoid this the equipment's can be embedded with the capability of the connecting to the internet to transfer the reports diagnosed, immediately to the concerned doctor and the patient over the internet. This would reduce the patient waiting time, vexation as well as the misplacing of the records avoiding the wrong treatment. The section below present the review of such IOT enabled medical imaging equipment's that makes the monitoring of the patient, diagnosis of the disease and the management of the patients record easy.

\section{IOT INVOLVEMENT IN MEDICAL IMAGING TECHNOLOGY}

Krishna, et al [1], the author proposes an "FPGA based computer aided diagram, internet of things enabled portable ultrasound imaging system for diagnosing the abnormalities in the kidney. The system architecture includes a wireless unit in it to transfer the diagnosis over the internet to the cloud for the storage and the further process. Chiuchisan et al [2] the real time configurable medical imaging equipment based on the Verilog is utilized to have enhanced medical images that are easily understandable $\mathrm{Lu}$ et al [3] the paper illustrates the "technology of the IOT utilized in the medical field, in all its areas such as the management, monitoring, medical imaging, in

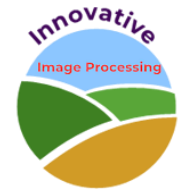


Journal of Innovative Image Processing (JIIP) (2019)

Vol.01/ No. 01

Pages: 51-60

https://www.irojournals.com/iroiip/

DOI: https://doi.org/10.36548/jiip.2019.1.006

patient, out patient's database etc. and its efficiency in the providing the information simultaneously to the physician, patient and the radiologist. The fig. 6 below shows the IOT efficiency in concurrently delivering the report to the entire group and the patient involved in the diagnosing.

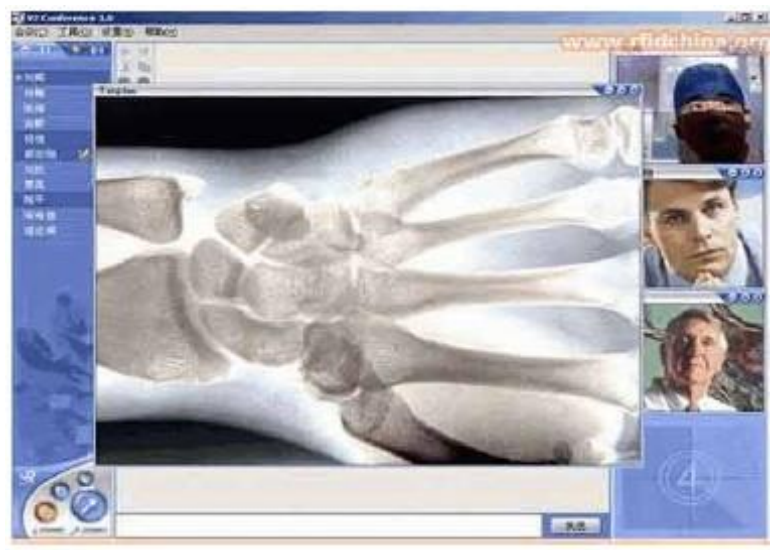

Fig .6 IOT enabled Medical Imaging [3]

Al-Majeed et al [4] a home based health care is made possible with a real time remote monitoring by integrating the internet of things with the medical systems. This would be very helpful for the physicians in monitoring the ECG, EEG etc. of the aged people without causing any weariness to them. Borovska, et al [5] the author presents the "experimental frame work of the investigating the importance of the internet of things in the medical imaging system. Hassanalieragh et al [7],Tyagi et al [8] and Plamenka, et al [9] they all elaborate the utilization of the IOT in the medical imaging to provide fast and the early diagnosis and the remote monitoring of the disease with the immediate suggestion on the precautions to be taken.

\section{SIGNIFICANCE AND THE LIMITATIONS OF THE IOT IN THE MEDICAL FIELD}

The internets of things are becoming more common in the medical field. The IOT in the health care industry fundamentally provides a "visualization of the material management, digitization of the medical information and the digitization of the medical process. The internet of things with the radio frequency identification ensures the eluding of the health care problems by supporting the production, distribution, and tracking of the medicines as well as the medical devices with the increase in the quality of the treatment and decrease in the cost of the management [14]. The incorporation of the RFID with the internet of things would enable multitude of advantages such as the

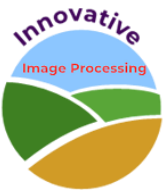


Journal of Innovative Image Processing (JIIP) (2019)

Vol.01/ No. 01

Pages: 51-60

https://www.irojournals.com/iroiip/

DOI: https://doi.org/10.36548/jiip.2019.1.006

pharmaceutical anti-counterfeiting, medical waste and emergency management, in the identification of the drug, medical record, and the medical equipment, drug storage etc. The IOT in the medical field enables the physicians to have an immediate monitoring of the disease progression and provide a real time suggestion and the precautions to be taken [1] it also allows a faster access of the patient data by the physician. The internet of things in the medical field enables us to have a smarter, healthier environment and a remote real time monitoring [3]. "The IOT enabled in the medical imaging systems provides a potential impact for the clinicians as well as the diagnostic approaches by enabling the individuals to understand the changes in the health in the real time"[5]. Despite the significance in the IOT enabled medical field, especially in the medical imaging there are certain limitations still prevailing in it causing difficulties in the real time monitoring. They are the "complications in the large scale dynamic networking and the node mobility management, data completeness, data compression and data security". So these limitations necessitates the "appropriate network mobility management methods and the network topology management structure" along with the perfect compression and the encryption algorithms to have an improved management, data compression and security respectively.

\section{CONCLUSION}

The paper presents the review of the IOT enabled medical imaging, with the detailed survey on the sources of the medical imaging along with the review of the IoT involvement in medical imaging technology. Further the paper provides the significance and the limitation in the IOT enabled medical imaging management and the monitoring, and also provides the countermeasure for the same. The paper projects that the IOT in the medical imaging would enhance the quality of the service and reduce the cost for the services along with the decrease in the vexation, weariness and the time consumption for the diagnosis of a disease. So the proper design with the perfect management, compression and the encryption algorithms would make the IOT enabled medical field efficient and successful. In future the paper is to continue with the survey on the available encryption algorithms to have a secure IOT enabled medical field with minimized hacking and misuse of the medical data.

\section{References}

[1] Krishna, K. Divya, V. Akkala, R. Bharath, P. Rajalakshmi, A. M. Mohammed, S. N. Merchant, and U. B. Desai. "Computer aided abnormality detection for kidney on FPGA based IoT enabled portable ultrasound imaging system." Irbm 37, no. 4 (2016): 189-197.

[2] Chiuchisan, Iuliana. "An approach to the Verilog-based system for medical image enhancement." In 2015 E-Health and Bioengineering Conference (EHB), pp. 1-4. IEEE, 2015. 
Journal of Innovative Image Processing (JIIP) (2019)

Vol.01/ No. 01

Pages: 51-60

https://www.irojournals.com/iroiip/

DOI: https://doi.org/10.36548/jiip.2019.1.006

[3] Lu, Dongxin, and Tao Liu. "The application of IOT in medical system." In 2011 IEEE International Symposium on IT in Medicine and Education, vol. 1, pp. 272-275. IEEE, 2011.

[4] Al-Majeed, Salah S., Intisar S. Al-Mejibli, and Jalal Karam. "Home telehealth by internet of things (IoT)." In 2015 IEEE 28th Canadian Conference on Electrical and Computer Engineering (CCECE), pp. 609-613. IEEE, 2015.

[5] Borovska, Plamenka, Desislava Ivanova, and Vladimir Kadurin. "Experimental Framework for the Investigations in Internet of Medical Imaging Things Ecosystem." QED 17 (2017): 20-21.

[6] Krishna, K. Divya, Vivek Akkala, R. Bharath, Pachamuthu Rajalakshmi, and Abdul Mateen Mohammed. "FPGA based preliminary CAD for kidney on IoT enabled portable ultrasound imaging system." In 2014 IEEE 16th International Conference on e-Health Networking, Applications and Services (Healthcom), pp. 257-261. IEEE, 2014.

[7] Hassanalieragh, Moeen, Alex Page, Tolga Soyata, Gaurav Sharma, Mehmet Aktas, Gonzalo Mateos, Burak Kantarci, and Silvana Andreescu. "Health monitoring and management using Internet-of-Things (IoT) sensing with cloud-based processing: Opportunities and challenges." In 2015 IEEE International Conference on Services Computing, pp. 285-292. IEEE, 2015.

[8] Tyagi, Sapna, Amit Agarwal, and Piyush Maheshwari. "A conceptual framework for IoT-based healthcare system using cloud computing." In 2016 6th International Conference-Cloud System and Big Data Engineering (Confluence), pp. 503-507. IEEE, 2016.

[9] Borovska, Plamenka, Desislava Ivanova, and Ivo Draganov. "Internet of Medical Imaging Things and Analytics in Support of Precision Medicine for the Case Study of Thyroid Cancer Early Diagnostics." Serdica Journal of Computing, Bulgarian Academy of Sciences, Institute of Mathematics and Informatics, accepted paper.

[10] Kieffer, J. C., A. Krol, Z. Jiang, C. C. Chamberlain, E. Scalzetti, and Z. Ichalalene. "Future of laser-based X-ray sources for medical imaging." Applied Physics B 74, no. 1 (2002): s75-s81.

[11]Nikolov, Svetoslav, and Joergen Arendt Jensen. "Virtual ultrasound sources in high-resolution ultrasound imaging." In Medical Imaging 2002: Ultrasonic Imaging and Signal Processing, vol. 4687, pp. 395-405. International Society for Optics and Photonics, 2002.

[12] Khoo, Vincent S., David P. Dearnaley, David J. Finnigan, Anwar Padhani, Steven F. Tanner, and Martin O. Leach. "Magnetic resonance imaging (MRI): considerations and applications in radiotherapy treatment planning." Radiotherapy and Oncology 42, no. 1 (1997): 1-15.

[13]Zinreich, Simion J., David W. Kennedy, Arthur E. Rosenbaum, B. W. Gayler, Ashok J. Kumar, and H. Stammberger. "Paranasal sinuses: CT imaging requirements for endoscopic surgery." Radiology 163, no. 3 (1987): 769-775.

[14] https://dzone.com/articles/applications-of-the-internet-of-things-in-the-medi-1

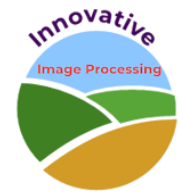


Journal of Innovative Image Processing (JIIP) (2019)

Vol.01/ No. 01

Pages: 51-60

https://www.irojournals.com/iroiip/

DOI: https://doi.org/10.36548/jiip.2019.1.006

[15] Kumar, R. Praveen, and S. Smys. "Analysis of dynamic topology wireless sensor networks for the Internet of Things (IOT)." Int. J. Innov. Eng. Technol.(IJIET) 8 (2017): 35-41.

[16] Kumar, R. Praveen, and S. Smys. "A novel report on architecture, protocols and applications in Internet of Things (IoT)." In 2018 2nd International Conference on Inventive Systems and Control (ICISC), pp. 11561161. IEEE, 2018.

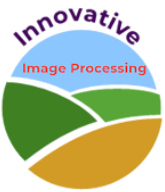

